\title{
Thermoelectricity for future sustainable energy technologies
}

\author{
Anke Weidenkaff $\left(^{*}\right)$ \\ Lehrstuhl für Chemische Materialsynthese, Institut für Materialwissenschaft \\ Universität Stuttgart - Heisenbergstraße 3, 70569 Stuttgart, Germany
}

\begin{abstract}
Summary. - Thermoelectricity is a general term for a number of effects describing the direct interconversion of heat and electricity. Thermoelectric devices are therefore promising, environmental-friendly alternatives to conventional power generators or cooling units. Since the mid-90s, research on thermoelectric properties and their applications has steadily increased. In the course of years, the development of high-temperature resistant TE materials and devices has emerged as one of the main areas of interest focusing both on basic research and practical applications. A wide range of innovative and cost-efficient material classes has been studied and their properties improved. This has also led to advances in synthesis and metrology. The paper starts out with thermoelectric history, basic effects underlying thermoelectric conversion and selected examples of application. The main part focuses on thermoelectric materials including an outline of the design rules, a review on the most common materials and the feasibility of improved future high-temperature thermoelectric converters.
\end{abstract}

\section{1. - Introduction}

Increasing efficiency of any kind of technical process is imperative from both an environmental and an economic standpoint. Since a large proportion (60-70\%) of the worldwide-applied energy carriers is converted into unused heat, recovery of at least part of this "waste" heat could lead to extensive savings. Thermoelectric (TE) converters,

(*) E-mail: weidenkaff@imw.uni-stuttgart.de 
which directly convert thermal energy into electric energy (Seebeck effect) appear particularly suitable for this purpose. They are all the more attractive due to their simplicity, silence and reliability due to the absence of moving parts. They also enable the direct and efficient conversion of abundant solar and geothermal heat. However, growth of thermoelectric conversion requires the development of effective, stable, sustainable and cost-efficient materials [1].

The collective term "thermoelectricity" summarizes various effects discovered almost 200 years ago. In 1822 the German-Baltic physicist and physician Thomas Johann Seebeck discovered an effect which was named after him [2]. Seebeck failed to recognize the actual cause of his findings and it was the Danish physicist and chemist Hans Christian Ørsted who called it a "thermoelectric effect" for the first time [3]. The Seebeck effect consists in a voltage gradient as the result of a thermal gradient across a sample. This is the consequence of a higher absolute value of the average velocity of charge carriers in the warm end of the sample, which leads to an effective diffusion towards the cold end. In a steady state this can be expressed by

$$
-\nabla U=S \nabla T
$$

where $U$ is the voltage, $S$ the Seebeck coefficient and $T$ the absolute temperature.

Twelve years later the French watchmaker, Jean Charles Athanase Peltier, observed the reverse process [4]. But just as Seebeck Peltier misinterpreted his results and it was not until 1838 that another German-Baltic physicist Heinrich Friedrich Emil Lenz realized the true nature of the Peltier effect and proved it to be an autonomous phenomenon [5]. It is defined as

$$
|\dot{Q}|=\Pi_{A B} \cdot I=\left(\Pi_{A}-\Pi_{B}\right) \cdot I,
$$

where $Q$ is the heat, $\Pi$ are the Peltier coefficients of the materials $A$ and $B$ and $I$ the electric current. Basically, the conditions of the Peltier effect are just the opposite of the Seebeck effect: in this case, an electric current induces heat transport, i.e. heat can be absorbed or released by applying a voltage.

Systematic studies on thermoelectricity were not further pursued until around 1885 . Between 1909 and 1911 the German physicist and mathematician Edmund Altenkirch succeeded in calculating the potential efficiency of thermoelectric generators and the performance of Peltier coolers [6,7] (fig. 1). Using his constant property model he already back then identified the parameters that make a good thermoelectric material: large Seebeck coefficient, high electrical conductivity (to minimize Joule heating due to electrical resistance) and low thermal conductivity (to minimize heat loss). In 1949 Ioffe developed the concept of the Figure of Merit, $Z$, describing the thermoelectric quality of a material which he presented in 1957 [8]:

$$
Z=\frac{S^{2} \sigma}{\kappa}
$$




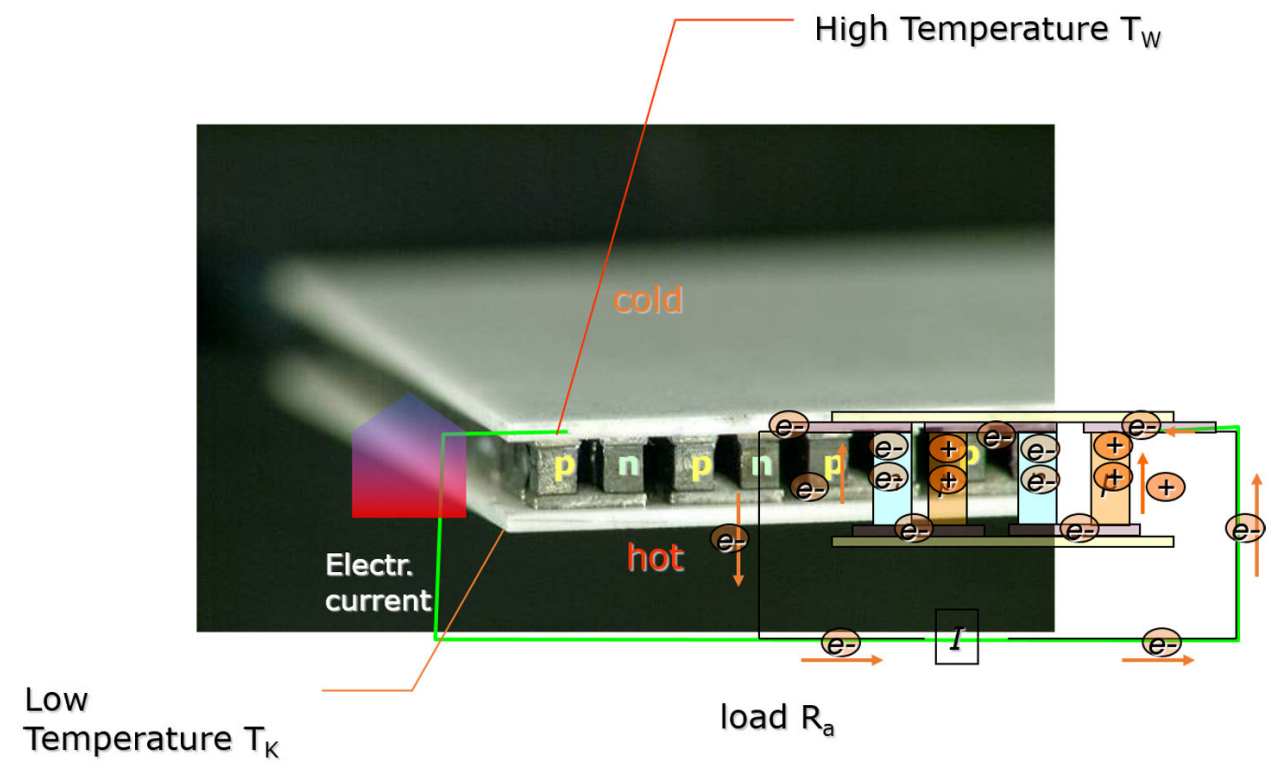

Fig. 1. - Thermoelectric generator (TEG) and Peltier cooler. The thermoelectric module is composed of an $n$-type and a $p$-type semiconducting material connected electrically in series through metallic electrical contact pads and thermally in parallel between ceramic ends.

where $S^{2} \sigma$ represents the power factor $P_{f}$, with $S$ the Seebeck coefficient, $\sigma$ the electrical conductivity and $\kappa$ the thermal conductivity.

Using the dimensionless product $Z T$,

$$
Z T=\frac{S^{2} \sigma}{\kappa} T
$$

the efficiency of a thermoelectric generator (TEG), i.e. the ratio between the energy delivered at the load and the heat energy absorbed at the hot side, can be expressed in terms of the Carnot-efficiency $\eta_{\text {Carnot }}$ (the upper efficiency limit of any heat engine) by

$$
\eta_{T E}=\frac{(1+Z T)^{1 / 2}-1}{(1+Z T)^{1 / 2}+\frac{T_{\text {cold }}}{T_{\text {hot }}}} \cdot \eta_{\text {Carnot }} .
$$

Figure 2 illustrates the relation between the maximum conversion efficiency and the $Z T$.

As mentioned above, eqs. (3) and (4) prove that suitable materials are characterized by a high Seebeck coefficient $|S|$, good electric conductivity $\sigma$ and low thermal conductivity $\kappa$. However, the selective modification of just one parameter is complicated by their mutual dependence. An increased electric conductivity usually comes along with an enhanced thermal conductivity; a higher carrier concentration improves the electric conduction at the expense of a reduced Seebeck coefficient. Thus, the main objective is 


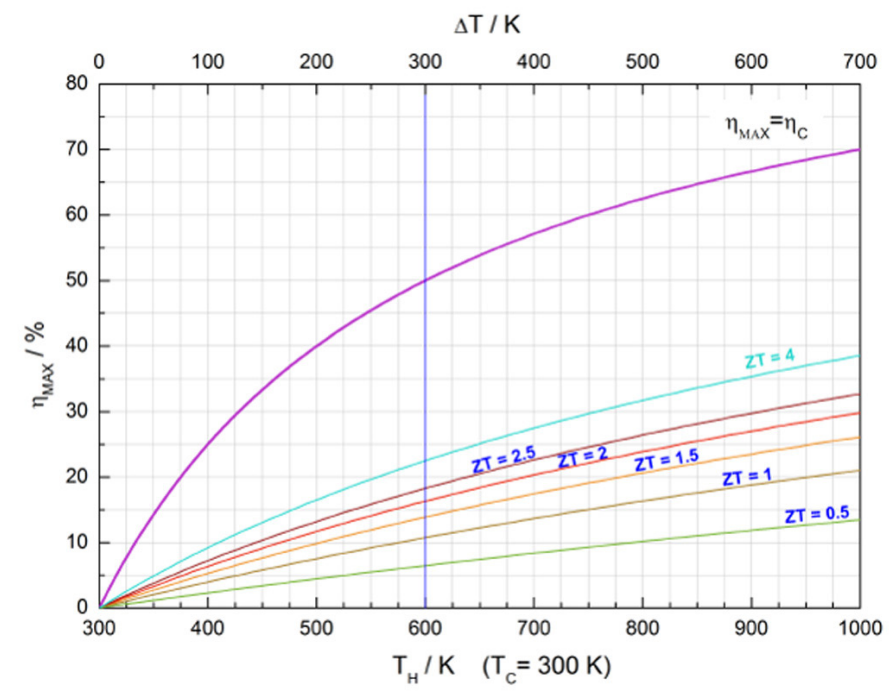

Fig. 2. - Relation between maximum conversion efficiency $\eta_{M A X}$ and the thermoelectric figure of merit $Z$.

to develop materials with a charge carrier concentration, mass and mobility providing a high power factor $P_{f}$ while at the same time suppressing the thermal conductivity (fig. 3). Moreover, it follows from eq. (4) that the economic fields of application arise in high-temperature environments.

The emergence of innovative synthesis and analysis methods together with the need of energy conservation resulted in a revival of thermoelectric research activities. Ioffe was one of the first to acknowledge the importance of alloying to reduce the thermal conductivity. Also, new nanostructuring techniques for the production of novel material classes with improved thermoelectric properties were developed. Since the mid-90s thermoelectric research has significantly increased. The focus is especially on high-temperature thermoelectrics and environmental-friendly and inexpensive materials.

\section{2. - Examples of applications}

Thermoelectric converters can be used for thermometry, power generation, heating and cooling purposes. The application generally requires $p$ - and $n$-type thermoelectrics. For thermometry, the resistance at the contact is coordinated with the temperature by calibrating against a reference thermometer. For power generation or heating / cooling devices, the thermoelements are connected electrically in series and sandwiched between a hot and a cold heat exchanging system. If a temperature gradient is applied the charge carriers travel along the $T$ gradient creating a current which can be used by a consumer. If a voltage is applied the same device can be used for heating and cooling, because heat is transported by the moving charge carriers. 


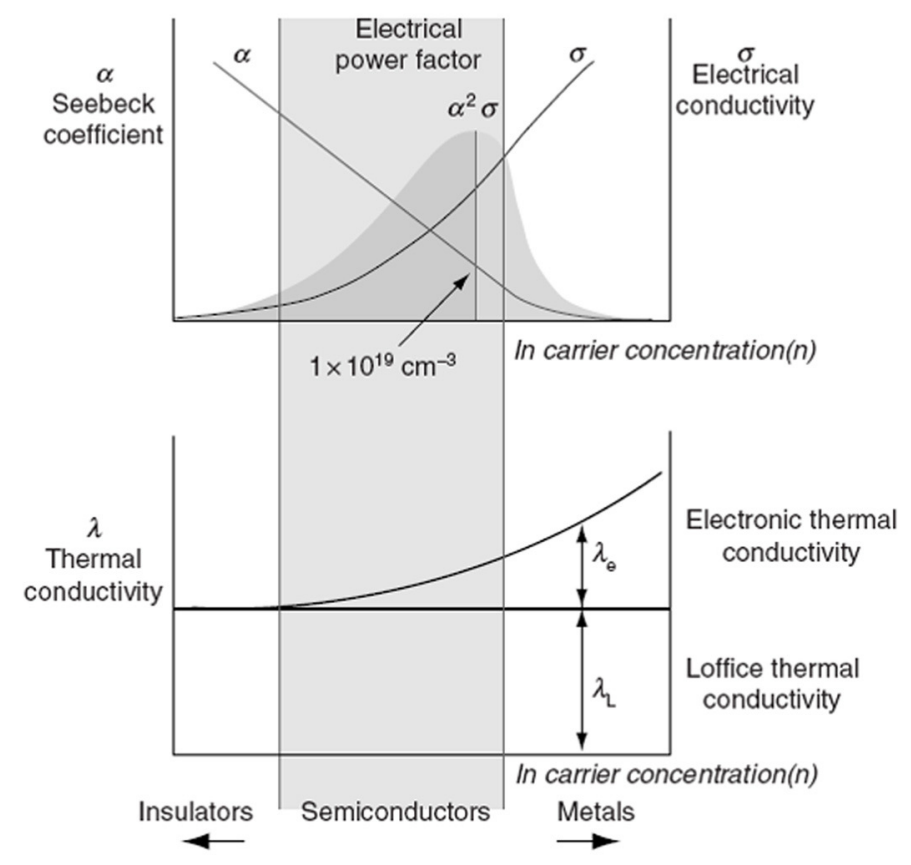

Fig. 3. - Schematic dependence of electrical conductivity, Seebeck coefficient, power factor and thermal conductivity on concentration of free carriers [9].

Wherever temperature gradients arise, thermoelectric devices can be applied. Owing to their simple design free of moving parts they offer durable and maintenance-friendly current supply. This can be of particular importance for unmanned remote facilities such as light and radio buoys, lighthouses, sensing stations for radar systems and pipeline monitoring equipment. An additional vast area of application is aerospace. Since the early 1960s, radioisotope thermoelectric generators (RTG) have been used as power supply for satellites and space crafts due to their longevity, reliability and small dimensions. They are the means of choice for missions to long for batteries or unfit for photovoltaics. Prominent examples include space probes of Pioneer, Voyager and Galileo missions and the power supply for scientific experiments by Apollo crews.

At present, application of this technique is largely confined to the above-mentioned niche products, since affordable and efficient TE materials are still lacking. The most significant improvements in performance are going to be expected at high temperature differences. Therefore, the key to a broader market would be inexpensive TE materials combining high conversion efficiency and adequate temperature stability.

A number of research projects have already demonstrated efficiency enhancements of technical installations by TEGs. In the project "Customized Hybrid Powertrains" (Cohyb), supported by Swisselectric (organisation of the Swiss electricity grid operators) together with the Swiss "Competence Center Energy and Mobility (CCEM)", TE recu- 


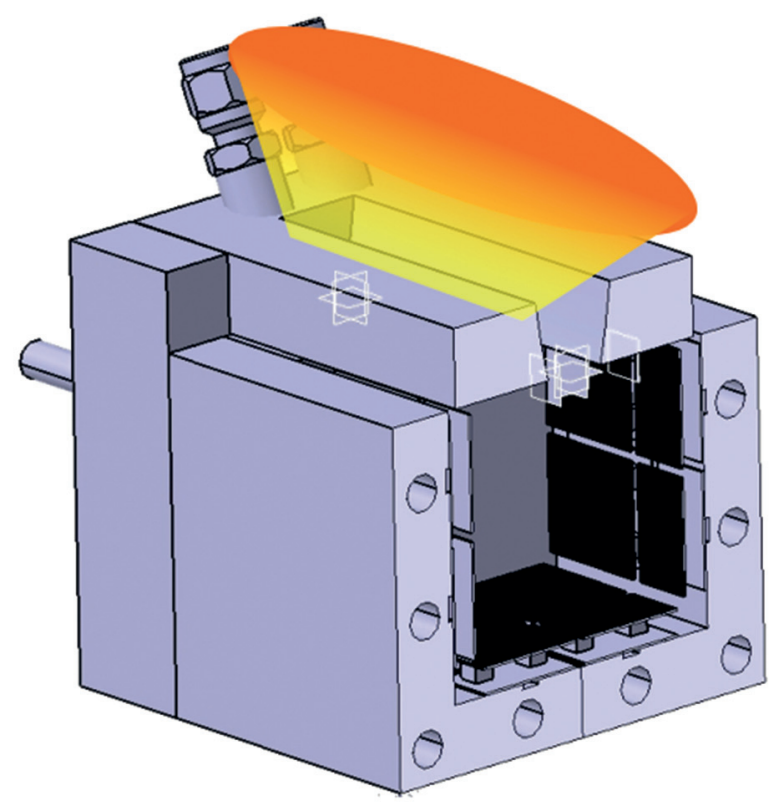

Fig. 4. - Solar thermoelectric cavity converter with 18 thermoelectric oxide modules (TOM) and K-type thermocouples for temperature monitoring.

peration of $\sim 120 \mathrm{~W}$ from engine exhaust heat, corresponding to $3 \%$ fuel saving, was achieved [10]. The goal of the project "High Temperature Thermoelectric Converter for Electricity Generation in a SOFC System" (HITTEC), cofounded by CCEM and the Swiss Federal Office of Energy (SFOE), was to develop a novel high-temperature (HT up to $1200 \mathrm{~K}$ ) thermoelectric converter (TEC) to be integrated, for instance, in a solid oxide fuel cell system (SOFC). The all-oxide module showed excellent HT-stability and a power output of $50 \mathrm{~W}$ at temperature differences of $\Delta T=400-500 \mathrm{~K}$ [11]. All-oxide modules where subject of research in another promising HT application. Thermoelectric conversion of simulated concentrated solar radiation was studied by means of a solar cavity converter (fig. 4).

The converter effectively captured the solar heat power and reduced re-radiation to $4 \%$. In this way the solar-to-electricity conversion efficiency could be increased by $62 \%$ compared to a module subjected to direct solar irradiation [12-14].

Subject to the development of good cost-benefit materials many more - particularly high-temperature - applications are conceivable. These include the conversion heat of geothermal or automotive origin or from machines of energy-intensive industries (steel, paper, glass, cement).

\section{3. - Materials}

3`1. Design rules. - Any effort to develop high-performance TE materials has to aim at an increasing $Z T$, i.e. a power factor $P_{f}\left(S^{2} \sigma\right)$ as high and a thermal conductivity $\kappa$ 
as low as possible (cf. eq. (4)). Beneficial effects on these parameters can be achieved by the following measures:

- Low thermal conductivity is a consequence of increased phonon scattering: This is achieved by mass fluctuation, heavy atoms, complex crystal structures or enhanced grain boundary and Umklapp scattering.

- A high Seebeck coefficient is associated with a steep rise of the density of states (DoS) at the Fermi level. This can be realized by tuning the band structure and band gap with low-dimensional systems, correlated electrons, low minority carriers or carriers with high effective mass.

- A good electrical conductivity relies on a high charge carrier concentration and mobility. This requires narrow-band-gap semiconductors and low electron scattering. Good electronic conductivity can be found in materials with strongly correlated electrons.

In addition, good temperature stability in air would be advantageous as the conversion efficiency is also a function of the temperature gradient.

Unfortunately, the complex physical relationships between these quantities illustrated by eqs. (6) to (8) lead to conflicting targets:

$$
\begin{aligned}
S & =\frac{8 \pi^{2} k_{B}^{2}}{3 e h^{2}} m^{*} T\left(\frac{\pi}{3 n}\right)^{2 / 3}, \\
\sigma & =n e \mu=\frac{n e^{2} \tau}{m^{*}}, \\
\kappa_{t o t} & =\kappa_{p h}+\kappa_{e l}=\kappa_{p h}+L \sigma T,
\end{aligned}
$$

where $k_{B}$ is the Boltzmann constant, $m^{*}$ the density of states effective mass, $h$ the Planck constant, $n$ the carrier concentration, $e$ the elementary charge, $\mu$ the carrier mobility, $\tau$ the relaxation time, $\kappa_{t o t}$ the total thermal conductivity, $\kappa_{p h}$ the phonon (or lattice) thermal conductivity, $\kappa_{e l}$ the electronical thermal conductivity, and $L$ the Lorenz number.

Equation (8) shows that lowering the phonon part $\kappa_{p h}$, whose influence usually outweighs that of the electronic part $\kappa_{e l}$, to values exhibited by glass-like structures would be the most potent way to reduce the overall thermal conductivity $\kappa_{t o t}$. For the phonon thermal conductivity $\kappa_{p h}$ one has

$$
\kappa_{p h} \propto C v_{s} l_{p h}
$$

where $C$ is the heat capacity per volume, $v_{s}$ the phonon velocity (speed of sound), and $l_{p h}$ the phonon mean free path. Therefore possible approaches towards increased phonon scattering are the generation of grain boundaries or defects $\left(\downarrow l_{p h}\right)$, complex crystal or "misfit" structures and using high-atomic-weight elements $\left(\downarrow v_{s}\right)$.

On the other hand, charge carrier mobility, i.e. the electric conductivity $\sigma$, increases with the crystallinity of the material. Thus, the challenge is to effectively scatter the 
phonons while preserving the electrical concuctivity. The compromise solution is the concept of a "phonon-glass electron-crystal" serving as a guideline for the design of thermoelectrics with improved properties [15].

What is more, the materials shall consist of inexpensive, abundant, and non-toxic elements.

3.2. Materials review. - Chalcogenides such as tellurides (e.g. $\mathrm{AgPb}_{18} \mathrm{SbTe}_{20}(\mathrm{LAST})$ ) are the currently best and most broadly applied thermoelectric materials. They operate in the low-temperature range up to maximum temperatures of ca. $500 \mathrm{~K}$ with reproducible $Z T$ values in the range of $0.7<Z T<1.5$. With a suitable nano-substructure, i.e. a $2 \mathrm{D}$ crystal structure, high power factors are common in e.g. $\mathrm{Bi}_{2} \mathrm{Te}_{3}$ [16] and $\mathrm{Bi}_{2} \mathrm{Te}_{3} / \mathrm{Sb}_{2} \mathrm{Te}_{3}$ superlattices [17]. Recently, sulfide-based minerals such as tetrahydrites (CuSb sulfides) have been considered alternative, low-cost candidates for thermoelectric power generation [18]. $\mathrm{TiS}_{2}$ nanosheets (sulphene) offer the possibility of a twodimensional confinement of electrons and phonons in favor of very high $Z T$ values [19].

Cage structures such as skutterudites or filled skutterudites (e.g. $\left.\mathrm{CeFe}_{4} \mathrm{Sb}_{12}\right)$ and $\mathrm{Si}$ or Ge-based clathrates reveal a complex crystal structure with "rattling" atoms and very low thermal conductivity $[20,21]$. They can be nanostructured by e.g. melt spinning or nano precipitation methods.

(Half)Heusler compounds are narrow-gap semiconductors (e.g. TiNiSn) with high effective mass charge carriers leading to large Seebeck coefficients of up to $S=400 \mu \mathrm{VK}^{-1}$ at room temperature. They are produced by co-melting the metals in an arc or induction furnace and a subsequent homogenation procedure. Ta substitution in these Heusler-based thermoelectrics suppresses the minority carriers (impurity band) and enhances the density-of-states effective mass $m_{C}{ }^{*}$ and the charge carrier mobility $\mu$ in $\mathrm{Zr}_{0.3} \mathrm{Hf}_{0.7-x} \mathrm{Ta}_{x} \mathrm{NiSn}[22,23]$. Nanostructuration can be achieved by spinodal decompositions, ball milling or nanoinclusions of full Heusler precipitates.

Oxides are stable in oxidizing environments (air) at high temperatures. Since the discovery of high Seebeck coefficient combined with low electrical resistivity of $\mathrm{NaCO}_{2} \mathrm{O}_{4}$ single crystals in the 1990s [24] at the latest, oxides have been recognized as a very important alternative material class for energy conversion in high-temperature environments. $\mathrm{NaCo}_{2} \mathrm{O}_{4}$ is a $p$-type cobalt oxide with a layered hexagonal structure. Materials with layered structures come close to a "phonon-glass-electron-crystal", the ideal configuration for most effective thermoelectric materials. The lower $2 \mathrm{D}$-arrangement of alternating layers is conducive both to phonon scattering and to charge carrier conduction and emphasizes the significance of the dimensionality for the development of innovative thermoelectric materials.

In addition, particular attention has been directed to transition metals-containing perovskite-type oxides owing their attractive properties and their non-poisonous and abundant elements [25-31]. Many ternary compounds with the general formula $\mathrm{ABX}_{3}$ adopt the perovskite structure, which is ideally made up of $\mathrm{BX}_{6}$ octahedra $(\mathrm{X}=\mathrm{O}, \mathrm{N})$ joined at corners with the A cation located in the center of eight octahedra (fig. 5). 


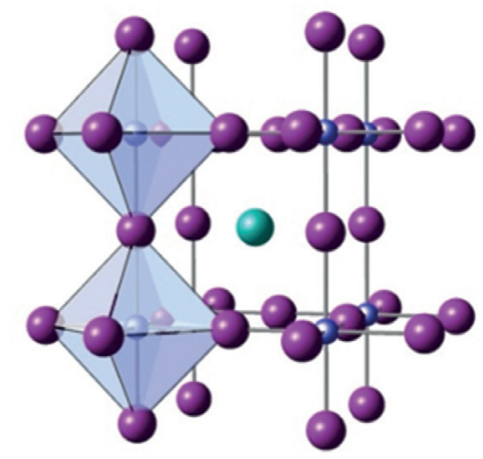

Fig. 5. - Ideal perovskite structure with each B cation (grey) surrounded by six anions (violet) forming an $\mathrm{BX}_{6}(\mathrm{X}=\mathrm{O}, \mathrm{N})$ coordination octahedron. The A cation (blue) is located in the center of eight octahedra.

The perovskite structure is very flexible in terms of chemical variations allowing adjustment of the band structure (DoS / band gap management) and physical properties (electronic correlation, spin entropy) by means of the chemical composition. Defects and local non-stoichiometry in the crystal structure are common and frequently tolerated leading to stable distorted structures, super structures, layered structures, polar structures, defects or non-stoichiometric (A-cation deficient, B-cation deficient, anion deficient) regenerative compounds. Perovskites show a wide variety of interesting properties, because many different compositions and also mixed-valence compounds can be stabilized in this flexible crystal structure. They have a particularly huge potential for many energy conversion processes due to their insulating, $p$ - or $n$-type semiconducting, metallic or even superconducting transport properties. Complex oxides with perovskite-type structures offer a suitable, correlated electrons system for improving the thermopower by spin-entropy contribution. Well-known and useful perovskites are cobaltates, titanates, manganates, niobates and tantalates.

Silicides such as, for example, $\mathrm{MnSi}_{1.75}$ and solid solutions of compounds based on $\mathrm{Mg}_{2} \mathrm{X}(\mathrm{X}=\mathrm{Si}, \mathrm{Ge}$, and $\mathrm{Sn})$ are environmentally friendly materials with high $Z T$ values and low weight [32].

Organic and hybrid materials are interesting for room temperature applications with a large surface area of the heat exchanger system. They are potentially low-cost and flexible materials. Conducting polymers including polyaniline, polypyrrol and poly $(3,4-$ ethylenedioxythiophene) (PEDOT) exhibit attractive thermoelectric properties and will be studied for thermoelectric applications [33].

New thermoelectric materials for energy conversion will provide an enhancement of the energy density, better thermal stability and efficiency of heat to electricity converters. The design of new materials is based on band gap, size and defect control by innovative synthesis processes. Furthermore, research on the large thermopower in correlated electronic systems and the low thermal conductivity due to grain boundaries hindering phonon transport has to be continued. Scalable, cost-efficient synthesis methods for envi- 
ronmentally friendly nanostructured materials and segmented converters using materials combinations will enable thermoelectric conversion at $T>700^{\circ} \mathrm{C}$ improving the power output.

\section{REFERENCES}

[1] Rowe D. M., "Thermoelectric Waste Heat Recovery as a renewable energy source", Int. J. Innov. Energy Syst. Power, 1 (2006) 13.

[2] Seebeck T., "Magnetische Polarisation der Metalle und Erze durch TemperaturDifferenz", Abh. K. Preuss. Akad. Wiss. Berlin (1822) 265.

[3] Ørsted H. C., "Nouvelles experiences de M. Seebeck sur les actions èlectro-magnetiques", Ann. Chim. Phys., 22 (1823) 375.

[4] Peltier J. C. A., "Nouvelles expériences sur la caloricité des courans électriques", Ann. Chim. Phys., 2 (1834) 371.

[5] Lenz E., "Einige Versuche im Gebiete des Galvanismus", Ann. Phys. (Leipzig), 120 (1838) 342, DOI: 10.1002/andp.18381200612.

[6] Altenkirch E., "Über den Nutzeffekt der Thermosäule", Phys. Z., 10 (1909) 560.

[7] Altenkirch E., "Elektrothermische Kälteerzeugung und reversible elektrische Heizung", Phys. Z., 12 (1911) 920.

[8] Ioffe A. F., Semiconductor Thermoelements and Thermoelectric Cooling (Infosearch, Ltd., London) 1957.

[9] Rowe D. M. (Editor), Thermoelectrics Handbook. Macro to Nano (CRC/Taylor \& Francis, Boca Raton, 2006).

[10] Guzzella L., "Cohyb - Customized Hybrid Powertrains", in CCEM Annual Activity Report 2013 (Competence Center Energy and Mobility (ccem.ch), Paul Scherrer Institut, Villigen (CH)) 2014, pp. 26-29.

[11] Heel A. and Populoh S., "HITTEC - High Temperature Thermoelectric Converter for Electricity Generation in a SOFC System", in CCEM Annual Activity Report 2015 (Competence Center Energy and Mobility (ccem.ch), Paul Scherrer Institut, Villigen $(\mathrm{CH})$ ) 2016, pp. 17-19.

[12] Tomes P., Trottmann M., Suter C., Aguirre M. H., Steinfeld A., Haueter P. and Weidenkaff A., "Thermoelectric Oxide Modules (TOMs) for the Direct Conversion of Simulated Solar Radiation into Electrical Energy", Materials, 3 (2010) 2801, DOI: $10.3390 / \mathrm{ma3042801.}$

[13] Tomes P., Suter C., Trottmann M., Steinfeld A. and Weidenkaff A., "Thermoelectric oxide modules tested in a solar cavity-receiver", J. Mater. Res., 26 (2011) 1975, DOI: $10.1557 / \mathrm{jmr} .2011 .125$.

[14] Suter C., Tomeš P., Weidenkaff A. and Steinfeld A., "A solar cavity-receiver packed with an array of thermoelectric converter modules", Sol. Energy, 85 (2011) 1511, DOI: 10.1016/j.solener.2011.04.008.

[15] Slack G., "New Materials and Performance Limits for Thermoelectric Cooling", in CRC Handbook of Thermoelectrics/CRC handbook of thermoelectrics, edited by RowE D. M. (CRC Press, Boca Raton, FL) 1995, pp. 407-440.

[16] Xie W., He J., Kang H. J., Tang X., Zhu S., Laver M., Wang S., Copley J. R. D., Brown C. M., Zhang Q. and Tritt T. M., "Identifying the specific nanostructures responsible for the high thermoelectric performance of (Bi,Sb)2Te3 nanocomposites", Nano Lett., 10 (2010) 3283.

[17] Venkatasubramanian R., Siivola E., Colpitts T. and O'Quinn B., "Thin-film thermoelectric devices with high room-temperature figures of merit", Nature, 413 (2001) 597, DOI: 10.1038/35098012. 
[18] LU X. and Morelli D. T., "Rapid synthesis of high-performance thermoelectric materials directly from natural mineral tetrahedrite", Magn. Res. Chem., 3 (2013) 129, DOI: $10.1557 / \mathrm{mrc} .2013 .26$.

[19] Zhang R.-Z., Wan C.-L., Wang Y.-F. and Koumoto K., "Titanium sulphene: twodimensional confinement of electrons and phonons giving rise to improved thermoelectric performance", Phys. Chem. Chem. Phys., 14 (2012) 15641, DOI: 10.1039/c2cp42949g.

[20] Tritt T. M. and Subramanian M. A., "Thermoelectric Materials, Phenomena, and Applications: A Bird's Eye View", MRS Bull., 31 (2006) 188, DOI: 10.1557/mrs2006.44.

[21] Eilertsen J., Surace Y., Balog S., Sagarna L., Rogl G., Horky J., Trottmann M., Rogl P., Subramanian M. A. and Weidenkaff A., "From Occupied Voids to Nanoprecipitates: Synthesis of Skutterudite Nanocomposites in situ", Z. Anorg. Allg. Chem., 641 (2015) 1495, DOI: 10.1002/zaac.201500137.

[22] Gaeązka K., Populoh S., Xie W., Yoon S., Saucke G., Hulliger J. and WeidenkafF A., "Improved thermoelectric performance of (Zr0.3Hf0.7) NiSn half-Heusler compounds by Ta substitution", J. Appl. Phys., 115 (2014) 183704.

[23] Zeier W. G., Schmitt J., Hautier G., Aydemir U., Gibbs Z. M., Felser C. and SNYDER G. J., "Engineering half-Heusler thermoelectric materials using Zintl chemistry", Nat. Rev. Mater., 1 (2016) 16032, DOI: 10.1038/natrevmats.2016.32.

[24] Terasaki I., Sasago Y. and Uchinokura K., "Large thermoelectric power in NaCo2O4 single crystals", Phys. Rev. B, 56 (1997) R12685, DOI: 10.1103/PhysRevB.56.R12685.

[25] Terasaki I., Iwakawa M., Nakano T., Tsukuda A. and Kobayashi W., "Novel thermoelectric properties of complex transition-metal oxides", Dalton Trans., 39 (2010) 1005, DOI: 10.1039/B914661j.

[26] Hashimoto H., Kusunose T. and Sekino T., "Influence of ionic sizes of rare earths on thermoelectric properties of perovskite-type rare earth cobalt oxides $\mathrm{RCoO} 3(\mathrm{R}=\mathrm{Pr}, \mathrm{Nd}$, Tb, Dy)", J. Alloys Compd., 484 (2009) 246.

[27] Weidenkaff A., Robert R., Aguirre M. H., Bocher L., Lippert T. and Canulescu S., "Development of thermoelectric oxides for renewable energy conversion technologies", Renew. Energy, 33 (2008) 342, DOI: 10.1016/j.renene.2007.05.032.

[28] Weidenkaff A., Robert R., Aguirre M. H., Bocher L. and Schlapbach L., "Nanostructured thermoelectric oxides with low thermal conductivity", Phys. Status Solidi Rapid Res. Lett., 1 (2007) 247, DOI: 10.1002/pssr.200701185.

[29] Koumoto K., Terasaki I. and Funahashi R., "Complex Oxide Materials for Potential Thermoelectric Applications", MRS Bull., 31 (2006) 206.

[30] Bocher L., Aguirre M. H., Logvinovich D., Shkabko A., Robert R., Trottmann M. and Weidenkaff A., "CaMn ${ }_{(1-x)} \mathrm{Nb}_{(x)} \mathrm{O}_{3}(x<$ or $=0.08)$ perovskite-type phases as promising new high-temperature n-type thermoelectric materials", Inorg. Chem., 47 (2008) 8077, DOI: 10.1021/ic800463s.

[31] Maignan A., Hébert S., Pi L., Pelloquin D., Martin C., Michel C., Hervieu M. and Raveau B., "Perovskite manganites and layered cobaltites: potential materials for thermoelectric applications", Cryst. Eng., 5 (2002) 365, DOI: 10.1016/S14630184(02)00048-5.

[32] Fedorov M. I. and Zaitsev V. K., "Thermoelectrics of Transition Metal Silicides", in Thermoelectrics Handbook: Macro to Nano, edited by Rowe D. M. (CRC/Taylor \& Francis, Boca Raton) 2006, pp. 31/1-31/19.

[33] Bubnova O., Khan Z. U., Malti A., Braun S., Fahlman M., Berggren M. and CRISPIN X., "Optimization of the thermoelectric figure of merit in the conducting polymer poly(3,4-ethylenedioxythiophene)", Nat. Mater., 10 (2011) 429, DOI: 10.1038/nmat3012. 